\section{Triglyceride concentration and coronary heart disease}

\section{High concentrations need treatment}

EdrToR,-Alan M Garber and Andrew L Avins state that use of the triglyceride concentration to guide lipid lowering treatment "must still be seen as experimental." I suggest that measurement of fasting plasma triglyceride concentrations should be mandatory in any patient with hypercholesterolaemia, particularly if lipid lowering treatment (either diet or drugs) is contemplated. The type of diet or drug to which a patient responds best depends on the relative amounts of circulating cholesterol and triglyceride. Moreover, patients with very high triglyceride concentrations (almos all of whom will have hypercholesterolaemia) should be identified and treated appropriately. They are at risk of pancreatitis, and neither the hypercholesterolaemia nor the hypertriglyceridaemia is likely to respond to conventional cholesterol lowering dietary or drug treatment.

A M CRUICKSHANK Consultant chemical pathologis

Department of Biochemistry,

Southern General Hospital NHS Trust,

Glasgow G51 4TF

1 Garber AM, Avins AL. Triglyceride concentration and coronary heart disease. $B M f$ 1994;309:2-3. (2 July.)

\section{Routine measurement is justified}

EDrToR,-In their editorial on triglyceride concentrations and coronary heart disease Alan $M$ Garber and Andrew L Avins focus on an insurmountable difficulty encountered when limited epidemiological tools are used in an attempt to disentangle biochemical variables. ${ }^{\prime}$ They conclude that measurement of triglyceride concentrations for screening and to guide treatment on the basis of the cardiovascular risk is experimental and less convenient than measurement of high density lipoprotein cholesterol concentrations. Serum cholesterol, triglycerides, and high density lipoprotein cholesterol are, however, related components of a complex system of lipoprotein transport, and a purist mathematical approach that takes little account of current clinical and laboratory practice is inappropriate. Although not central to my concern with the views of Garber and Avins, there is good evidence for an independent association between triglyceride concentrations and coronary heart disease which corrects for high density lipoprotein cholesterol concentration. ${ }^{2}$ This is particularly notable in diabetes mellitus. ${ }^{34}$

The concern that the benefits of treatment of hypertriglyceridaemia on coronary heart disease may not be independent of the high density lipoprotein cholesterol concentration falls foul of the same difficulty in separating out their predictive value: it is the combination of hypercholesterolaemia, hypertriglyceridaemia, and low high density lipoprotein cholesterol concentration that marks out people as more likely to benefit from treatment. Failure to measure the serum triglyceride concentration could result in an underestimation of risk, particularly in women with modest hypercholesterolaemia $(6 \cdot 5-8 \cdot 0 \mathrm{mmol} / \mathrm{l})$. High density lipoprotein cholesterol concentrations may be lower than $0.9 \mathrm{mmol} / 1$ in $0.5 \%$ of such cases, ${ }^{5}$ and the presence of hypertension and diabetes increases the relevance of hypertriglyceridaemia, whose prevalence may be 10-20 times higher. ${ }^{\circ}$

The suggestion by Garber and Avins that measurement of high density lipoprotein cholesterol concentration is more convenient than measurement of triglyceride concentration takes no account of current laboratory practice. Measurement of high density lipoprotein cholesterol may not be routinely available in up to
$30 \%$ of biochemistry laboratories in Britain. ${ }^{7}$ Serum triglycerides can be measured simultaneously with serum cholesterol, with the same analyser. The additional costs of staff and reagents for measurement of serum triglyceride is $10 p$, compared with $£ 1.50$ for measurement of high density lipoprotein cholesterol. Furthermore, national quality control schemes exist for estimation of triglyceride but not high density lipoprotein cholesterol, which is isolated by different techniques. Measurement of both triglyceride and high density lipoprotein cholesterol concentrations also allows estimation of low density lipoprotein cholesterol concentrations without preparative ultracentrifugation.

I suggest that routine measurement of (fasting) serum cholesterol and triglyceride concentrations is appropriate in the screening of patients at risk of coronary heart disease, with subsequent confirmation of hyperlipidaemia at least once. Measurement of high density lipoprotein cholesterol concentration is more appropriate on a single occasion before drug treatment is considered.

Directorate of Medicine,

PETER H WINOCOUR

Queen Elizabeth II Hospita

Welwyn Garden City,

Hertfordshire AL7 4HQ

1 Garber AM, Avins A L. Triglyceride concentration and coronary heart disease. $B M F$ 1994;309:2-3. (2 July.)

2 Bainton D, Miller NE, Bolton CH, Yarnell JWG. Plasma triglyceride and high density lipoprotein cholesterol as predictors of ischaemic heart disease in British men. Br Heart $\mathcal{F}$ 1992;68:60-6.

3 Uusitupa MIJ, Niskanen LK, Siitonen O, Voutilainen E, Pyorala $K$. Ten-year cardiovascular mortality in relation to risk factors and abnormalities in lipoprotein composition in type 2 (noninsulin-dependent) diabetic and non-diabetic subjects. Diabetologia 1993;36:1175-84.

4 Winocour PH, Durrington PN, Bhatnagar D, Mbewu AD, Ishola $M$, Mackness $M$, et al. A cross-sectional evaluation of cardiovascular risk factors in coronary heart disease associated with type 1 (inslulin-dependent) diabetes mellitus. Diabetes Res Clin Pract 1992;18:173-84.

5 Neil HAW, Mant D, Jones L, Morgan B, Mann JI. Lipid screening: is it enough to measure total cholesterol concentration? BMf 1990;301:584-7.

6 Harland JOE, Winocour PH, Ramaiya K, Brown L, Kalluvya S, Millar JP, et al. Detection of clustering of cardiovascular risk factors during a single practice-based screening programme. Cardiovascular Risk Factors 1994;4:18-27.

7 Laker MF, Reckless JPD, Betteridge DJ, Durrington PN, Miller JP, Nicholls DP, et al. Laboratory facilities for investigating lipid disorders in the United Kingdom: results of the British Hyperlipidaemia Association survey. I Clin Pathol 1992;45: 102-5.

\section{Community geriatricians}

EDrToR,-As one of the few consultant community physicians in geriatric medicine, I wish to comment on correspondents' criticisms of Jackie Morris's suggestion that posts for consultant geriatricians with a community orientation should be created. ${ }^{12}$ Morris's suggestion was not at odds with the recommendation by the British Geriatrics Society that one or more consultants in a hospital department should take a lead role in developing community initiatives. Geriatrics is a broad based specialty. Many geriatricians have commitments to acute medicine or an interest in a subspecialty such as neurological or orthopaedic rehabilitation, which may prevent them spending much time on community activities. Surely it is legitimate for some geriatricians to concentrate on developing community initiatives as a special interest. I agree that such specialists should be full members of the department of geriatrics, undertaking all aspects of hospital based work as well as community activities.

I understand David Black's concern that posts for community geriatricians may be created with the underlying aim of restricting acute hospital care for frail elderly people. ${ }^{1}$ This is unlikely to happen if posts are created as described above. Indeed, in Newcastle our experience is just the opposite. In our work with social services, providing specialist input into community care assessments, we see people who would not normally present to the geriatric service (for various reasons), and our involvement ensures that more, not fewer, frail old people gain access to specialist acute or rehabilitation facilities. With the current emphasis on community care and the shift to primary care services I am sure that this aspect of our work will be increasingly important.

I do not share Black's fear that it will be difficult to find applicants of suitable calibre to fill these posts. As with any new development, there will be a lag phase while sufficient interested people receive appropriate training, but the framework for training is already available, ${ }^{3}$ and it should not be difficult to ensure that trainees receive the requisite experience. Increasingly, trainees express an interest in my post, which they see as a natural development in the context of today's health service.

Of course, community geriatricians should not duplicate or supplant primary care services but should complement and enhance them. General practitioners seem to welcome the extra support I provide. I believe that we are working in partnership to ensure the highest quality service for all elderly people under our care. It would be unfortunate if unnecessary division was created between professionals because of misunderstandings or suspicion about the post of community geriatrician.

GABRIELLE GREVESON Consultant community physician in geriatric medicine Department of Medicine for the Elderly,

Newcastle General Hospital, Newcastle upon Tyne NE4 6BE

1 Correspondence. Community geriatricians. BMf 1994;309 127-8.

Morris J. The case for the community geriatricians. $B M f$ 1994;308:1184. (7 May.)

3 Swift CG, James OFW. Training and manpower in geriatric medicine. London: British Geriatrics Society, 1991.

\section{Vitamin $\mathrm{K}$ for neonates}

EDITOR,-Jim Slattery commits a serious error in suggesting that breastfeeding mothers who experience major difficulties during the early weeks should give their babies supplementary formula milk.' This is bad advice and contrary to the results of research on establishing breast feeding. Breastfeeding mothers who experience difficulties need individualised help from their midwife or health visitor without delay. Breastfeeding counsellors are also available to give support and information of a non-medical nature. All health care workers should remember that formula milk is bad for breast feeding. We were heartened by Gerald Draper and Andrew McNinch's statement that "while we continue to debate vitamin $\mathrm{K}$ regimens we should be unanimous in promoting breast feeding." 2

The National Childbirth Trust recently asked members to send details of policies and practices on administration of vitamin $\mathrm{K}$. We found that when written material is available for parents there is a danger that it may be taken to imply that formula feeds are preferable to breast milk since they contain more vitamin $\mathrm{K}$. The many benefits of breast feeding, such as a reduction in the number of infections, are not generally mentioned. This can be a deciding factor for mothers who are not fully committed to breast feeding. The Health Visitors Association notes that "the confusion is causing a reduction in those wishing to or continuing to breast feed."

The concentration of vitamin $\mathrm{K}$ in colostrum is higher than that in mature milk, and the concentration in hindmilk is 1.9 times that in foremilk. ${ }^{3}$ Unrestricted breast feeding, with the baby well positioned at the breast, will maximise the baby's 
intake of hindmilk and hence of vitamin $\mathrm{K} .{ }^{4}$ If the baby needs extra vitamin $\mathrm{K}$ it can be given as a separate supplement.

Slattery makes some cogent points about the ethics of routine treatment of babies with vitamin $\mathrm{K}$ in the absence of controlled trials looking at possible side effects. ${ }^{15}$ Interpreting statistics on haemorrhagic disease of the newborn in relation to feeding and prophylactic treatment is extremely difficult. In the leaflets we have collected the incidence of haemorrhagic disease quoted to parents varies from 1 in 50 to 10 in 1.7 million babies. We welcome the developing debate on the size of the problem and the benefits and risks of the alternative preventive strategies. In particular, we value discussion about the appropriateness of a randomised controlled trial.

Despite the practical difficulties and the long wait for results ${ }^{2}$ we believe that a trial is appropriate, given that the whole population of newborn babies is currently targeted. Careful attention should be paid to producing clear information based on research for all parents considering treatment for their child.

MARY NEWBURN Head of policy research ROSEMARY DODDS Policy research officer

National Childbirth Trust,

London W3 8AR

1 Slattery J. Vitamin K for neonates. BMf 1994;308:1635. (18

2 June.) BMF 1994;308:867-8. (2 April.)

3 Von Kries R, Shearer PT, McArthy M, Haug G, Harzer G, Gobel U. Vitamin $K_{1}$ content of maternal milk: influence of the stage
on U. Vitamin $K_{1}$ content of maternal milk: influence of the stage
of lactation, lipid composition, and vitamin $K$ supplemenof lactation, lipid composition, and vitamin K suppl
tation given to the mother. Pediatr Res 1987;22:513-7.

4 Woolridge $\mathrm{MW}$, Baum JD. Infant appetite-control and the regulation of breast milk supply. Children's Hospital Quarterly 1991;3:113-9.

5 Slattery J. Why we need a clinical trial for vitamin K. $B M f$ 1994;308:908-10. (2 April.)

\section{Treating hypertension after stroke}

EdToR,-Janice E O'Connell and Christopher S Gray's editorial highlights the uncertainty concerning the optimal management of hypertension in acute stroke. ${ }^{1}$ I disagree with the authors over the treatment of hypertension after intracerebra haemorrhage. They advocate treating high systemic pressures (systolic $>200 \mathrm{~mm} \mathrm{Hg}$; diastolic $>120 \mathrm{~mm} \mathrm{Hg}$ ) and quote a paper by Lavin in support of this. ${ }^{2}$ Lavin in fact suggested that intracranial pressure, rather than systemic blood pressure, should be reduced. Although he added that treatment of systemic hypertension may reduce cerebral oedema, he qualified this by suggesting that systemic hypertension should be reduced by no more than $20 \%$ and that ischaemia of the border zone may result. In response to a subsequent letter Lavin clarified that his personal bias was to lower intracranial pressure and not systemic blood pressure. ${ }^{3}$

A recent review of decisions on treatment in acute hypertension after stroke concluded that the role of hypotensive therapy in intracerebra haemorrhage remains unresolved and that, as with cerebral infarction, there are cogent reasons for believing that reduced systemic blood pressure may worsen cerebral blood flow, with resultant ischaemic damage. ${ }^{4}$

Although O'Connell and Gray state that no randomised trials looking specifically at antihypertensive treatment in acute stroke have been published, such a study has been carried out in ischaemic stroke. ${ }^{5}$ Although the study was small no clinical benefit was observed, but it was sug gested that nicardipine and possibly other calcium channel antagonists may impair cerebral blood flow.
I believe that, until robust evidence exists to support the use of antihypertensive drugs in acute stroke (either ischaemic or haemorrhagic) in adults, these drugs should be withheld initially unless hypertensive encephalopathy or aortic dissection is present.

\section{RICHARD J DAVENPORT} Clinical research fellow

Neurosciences Trials Unit,

Department of Clinical Neurosciences,

University of Edinburgh,

Edinburgh EH4 2XU

1 O'Connell JE, Gray CS. Treating hypertension after stroke. BMF 1994;308:1523-24. (11 June.)

2 Lavin P. Management of hypertension in patients with acute stroke. Arch Intern Med 1986;146:66-8.

Lavin P. Sodium nitroprusside treatment in patients with acute

strokes. Arch Intern Med 1986;146:1454.
4 Powers WJ. Acute hypertension after stroke: the scientific basis for treatment decisions. Neurology 1993;43:461-7.

5 Lisk DR, Grotta JC, Lamki LM, Tran HD, Taylor JW, Molony $\mathrm{DA}$, et al. Should hypertension be treated after acute stroke? A randomized controlled trial using single photon emission computed tomography. Arch Neurol 1993;50:855-62.

\section{Running an emergency helpline}

\section{The media may exploit doctors}

EDITOR,-In their article on how to run an emergency helpline Cameron Stark and colleagues state, "If information can be adequately transmitted by the media a helpline should not be needed." Our experience is that dealing with the media is not that simple.

We recently conducted a look back exercise after learning that a health care worker at a local hospital was positive for HIV. We had wanted to avoid unhelpful and prejudicial publicity caused by insensitive handling of the subject by the media, and so we avoided contact with the press for as long as possible. Patients who needed to be traced were approached personally through their general practitioners, and the tracing exercise was completed without press coverage.

The media inevitably heard of the incident. Unnecessary anxiety was generated among the general public, and a helpline was set up to answer concerns that the media raised. Over 2000 calls were received in the first 48 hours, and peaks of calls coincided with the broadcasting of news bulletins. The helpline proved a useful way of conveying information but was costly in terms of time and staff. There were also obvious conflicts of interest between us and the news hungry press, not the least of which concerned the confidentiality of the health care worker. Fortunately, we were able to maintain this.

The relationship between the health service and the press can be a valuable one, but once the media get hold of what they perceive to be a newsworthy health issue doctors need to make sure that they use the media to their advantage and are not exploited.

ALISON WALKER Registrar in public health NAUREEN BHATTI Senior registrar in public health MARK MCCARTHY Camden and Islington Health Authority, Director of public health London NW1 $12 \mathrm{LJ}$

1 Stark C, Christie P, Marr AC. How to run an emergency helpline. $B M f$ 1994;309:44-6. (2 July.)

\section{Guidance from NHS is required}

EDrToR,-Cameron Stark and colleagues' article on how to run an emergency helpline described the actions necessary once the decision has been taken to establish such a helpline. ${ }^{1}$ More pressing issues, however, are under what circumstances such helplines are warranted and what constitutes such a serious risk to the patient that a "look back" exercise is needed. Over the past year such exercises have taken place in relation to helplines set up to provide advice on HIV and hepatitis B virus infection and unreliable results of cervical smear tests. In many cases the risk to patients may be remote, and the risk should be considered against the cost of such exercises and their tendency to provoke media alarm and public confusion.

The NHS Executive has no coherent overall view on look back exercises and no guidance for health authorities and trusts on the criteria for undertaking such initiatives and setting up helplines. Such guidance is needed to prevent unnecessary alarm among patients and to ensure that resources are directed towards such exercises only when there is clear risk to a large population of patients.

J B L HOWELI Chairman

Board of Science and Education,

BMA,

London WC1H 9JP

1 Stark C, Christie P, Marr AC. How to run an emergency helpline. $B M f$ 1994;309:44-5. (2 July.)

\section{Training in psychosexual medicine}

EDrToR,-May Duddle's criticism of the Institute of Psychosexual Medicine's training is il informed' as she has taken no part in the institute's training, research, and development, which have been carried out nationwide for 30 years. I wish to set the record straight.

Doctors accepted for basic training must expect to meet patients who expect examination of their sexual organs as well as emotional understanding in general practice, patients come for family planning, genitourinary medicine, gynaecology, and andrology. Certain nurses, midwives, and obstetric physiotherapists also have to use intimate therapeutic techniques, and some join seminars with our accredited leaders. The clinical problems studied are thus wider than, though include, dysfunction in sexual performance. We expect our trainees to learn other techniques elsewhere: you would not expect to learn ear, nose, and throat surgery on an obstetric firm.

Training in seminars aims to convey a precise, extra psychosomatic skill (distinct from other nondirective verbal counselling and psychotherapy) to everyday consultations, whether they are for the overt presentation of sexual difficulty or marital discord; the fitting of a contraceptive or an intracavernosal injection; postmenopausal atrophy or a widower's impotence. In each case we examine less conscious aspects of the immediate doctor-patient interaction, considering what light they throw on other relationships. Secondly, we learn from the patients' reaction to, or anticipation of, genital examination something of their vision of their personal sexuality.

Duddle comments that we do not encourage partners to attend. Many young men with premature ejaculation or women with vaginismus are too anxious to be able to expose themselves to a partner, let alone to propose joining him or her in behaviour therapy. Furthermore, primary care doctors, unlike psychiatrists accepting referrals, must regard "the patient" as being whoever consults them. Much time in training concerns why doctors find themselves wishing to split up a couple who have elected to present together and conversely, why in some cases doctors send for a partner they have never met, rather than noticing that they have a complaining or blaming patient before them. ${ }^{2}$

Finally, as an experienced psychotherapist Duddle will surely agree that the psychodynamics 